\title{
Observation of asymmetric spectrum broadening induced by silver nanoparticles in a heavy-metal oxide glass
}

\author{
N. Zhavoronkov ${ }^{1}$, R. Driben ${ }^{2,3^{*}}$, B. A. Bregadiolli ${ }^{4}$, M. Nalin $^{4}$ and B. A. Malomed ${ }^{5}$ \\ ${ }^{1}$ Max-Born-Institut for Nonlinear Optics and Short Pulse Spectroscopy, Max-Born-Str. 2a, D-12489 Berlin, Germany \\ ${ }^{2}$ Institut fur Physik, Universitat Rostock, Universitatsplatz 3, 18055 Rostock, Germany \\ ${ }^{3}$ Jerusalem College of Engineering, Ramat Beit HaKerem, POB 3566, Jerusalem, 91035, Israel \\ ${ }^{4}$ LAVIE - Departament of Chemistry - UFSCar, \\ Rodovia Washington Luiz Km 235, 13565-905, São Carlos, SP, Brazil and \\ ${ }^{5}$ Department of Physical Electronics, School of Electrical Engineering, \\ Faculty of Engineering, Tel Aviv University, Tel Aviv 69978, Israel
}

(Dated: April 24, 2022)

\begin{abstract}
We demonstrate experimentally and support by a theoretical analysis an effect of asymmetric spectrum broadening, which results from doping of silver nanoparticles into a heavy-glass matrix, $90\left(0.5 \mathrm{WO}_{3}-0.3 \mathrm{SbPO}_{4}-0.2 \mathrm{PbO}\right)-10 \mathrm{AgCl}$. The strong dispersion of the effective nonlinear coefficient of the composite significantly influences the spectral broadening via the self-phase modulation, and leads to a blue upshift of the spectrum. Further extension of the spectrum towards shorter wavelengths is suppressed by a growing loss caused by the plasmon resonance in the silver particles. The red-edge spectral broadening is dominated by the stimulated Raman Scattering.
\end{abstract}

Keywords: plasmon; surface resonance; stimulated Raman scattering; nonlinearity dispersion

Dielectric composites containing metal nanoparticles (MNPs) have been intensively studied for the last two decades (see, e.g., Refs. 113), as they play a central role in the rapidly growing fields of nano-optics and plasmonics. The interaction between light and MNPs is dominated by charge-density oscillations on the closed surface of the particles, alias localized plasmon resonances, and leads to a strong field enhancement in the MNP's near field. To mention just a few applications of such systems, localized plasmons allow greatly increased signal strengths in the Raman spectroscopy and surface spectroscopy, enabling the detection of single molecules [4, significant enhancement of the emission rate of fluorescent molecules and quantum dots [5], and the direct generation of high harmonics by nJ pulses produced by a laser oscillator, without the use of amplifiers [6]. The property of MNPs which is most important for applications to nonlinear optics is their large intrinsic nonlinearity coefficient. Far from the plasmon resonance, the third-order susceptibility of silver MNPs in the nanoparticle composite is about seven orders of magnitude higher than in the host material [7-11]. Since the local electric field in MNPs is enhanced at the plasmonic resonance, the nonlinear response of the metal can be additionally significantly amplified as the wavelength shifts towards the resonance.

The generation of coherent white light (or supercontinuum generation) in microstructured fibers by fs and ps pulses has attracted a great deal of attention and found many applications (see review [12]). Recently, much effort has been devoted to decrease the power or energy threshold necessary to generate octave-spanning spectra by reducing the relevant diameters to the submicron scale 13] or using nanowires made of materials with high nonlinearities [14. The reduction of the power threshold is particularly important for the realization of cost-effective and compact SC sources. An alternative approach to lowering the threshold condition for nonlinear processes is the utilization of composites containing MNPs. Previously, white-light generation in nanometerscale antennas related to photoluminescence [15], and in colloidal MNP systems synthesized by means of ablation [16] were reported. Additionally, a relatively small spectral broadening in aqueous colloids with very low filling factor was observed [17. In recent works, a mechanism of the wavelength-dependent nonlinearity in aqueous colloids [17, 18, and in silica glasses volume-doped by silver MNPs, was studied, and it was predicted that it can lead to the generation of supercontinuum [17, 19 and solitons 20. In the present Letter we produce an experimental evidence of asymmetric spectral broadening due to the presence of nanoparticles in composites based on $\mathrm{WO}_{3}$ host glass, and support these observations by extended numerical calculations.

The synthesis of the glasses was carried out by melting 99\%-grade-purity materials $\mathrm{WO}_{3}, \mathrm{PbO}, \mathrm{AgCl}$ from Aldrich, and $\mathrm{SbPO}_{4}$ prepared as in Ref. 21] in a platinum crucible kept in an electrical furnace during 30 minutes at $1100^{\circ} \mathrm{C}$. After homogenization, the melt was cooled to $950^{\circ} \mathrm{C}$, left at that temperature for 5 minutes, and then cast into a brass mold, pre-heated to $400^{\circ} \mathrm{C}$. Glass pieces were left for annealing at that temperature during four hours to reduce thermal stresses, and then the furnace was turned off and cooled to the room temperature under its natural cooling rate. Silver MNPs were implanted by heat treatment of the glass sample under normal pressure for two hours at $450^{\circ} \mathrm{C}$.

The size distribution and shape of silver MNPs have been determined by means of the transmission electron microscopy (TEM). The particles demonstrate a quasi- 
spherical shape, as it is can be observed in the TEM image in the inset to Fig. 1(a). The result of the MNP analysis for the size distribution is presented in Fig. 11(a), giving the mean diameter around $6 \mathrm{~nm}$. The absorption spectrum of the annealed sample in Fig.1 (b) demonstrates a new absorption shoulder at $\sim 515 \mathrm{~nm}$, in comparison to the "as prepared" glass composition, which is attributed to the surface plasmon resonance of silver. The strong red-shift of the resonance in this case is accounted for by the high linear refractive index of glass matrix, $n \approx 2.11$.
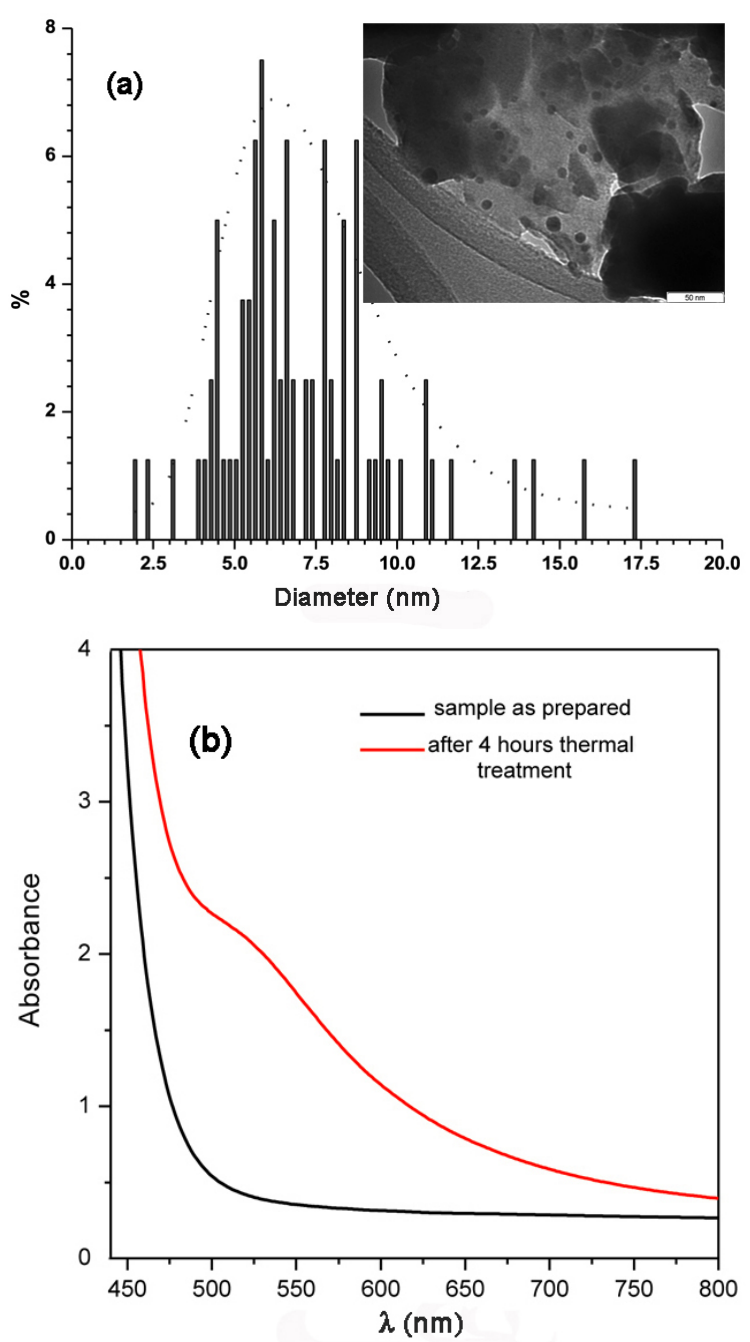

FIG. 1: (Color online) (a) Size distribution silver nanoparticles determined by transmission electron microscopy (TEM). A quasi-spherical geometry as demonstrated in TEM image (inset). (b) The absorption spectrum of the annealed sample.

A Ti:sapphire-based laser system, generating 30-fs pulses centered at $800 \mathrm{~nm}$ with up to $3 \mathrm{~mJ}$ energy, was used in the experiment. The spatial profile of the output beam was measured to be Gaussian with quality parameter $M^{2}$ close to 1 . The diameter of the laser beam was reduced with a use of a 1:3 telescope to $5 \mathrm{~mm}$ at the $1 / \mathrm{e}$ level. The beam's power was attenuated by a factor of 10 with the help of a rotating optical chopper, to avoid thermal effects in the samples. The $2 \mathrm{~mm}$ thick $\mathrm{WO}_{3}$ glass samples, both doped with the silver MNPs and pure ones, were placed between a pair of mirrors, introducing dispersion of $-40 \mathrm{fs}^{2}$ per bounce, to compensate the material dispersion of the glass matrix and prevent the distortion of the pulse in the temporal domain. The mirror-pair was also used to arrange additional passes of the pulses through the samples, by adjusting the relative position of the mirrors. It was not reasonable to perform more that four passes through the silver-doped sample because of a relative low single-pass transmissivity of $70 \%$, which is mostly determined by high losses due to the reflection from uncoated glass surfaces, with the respective refractive index $n>2$, and by imperfections of the surfaces. The spectrum of the transmitted beam was measured using an intensity-calibrated spectrometer (AvaSpec-2048 190-1100 nm), after attenuating the Fresnel reflection from the fused silica edge.

If both the average distance between the MNPs and their size are much smaller than the wavelength of light, the effective-medium description, based on the MaxwellGarnett model, can be applied to spherical particles with uncorrelated positions, up to relatively large values of the filling factor, $f \lesssim 0.2$. In this model the effective dielectric constant of the composite is given by $\epsilon_{\mathrm{eff}}=$ $\epsilon_{h}(1+2 \sigma f) /(1-\sigma f)$ with $\sigma \equiv\left(\epsilon_{i}-\epsilon_{h}\right) /\left(\epsilon_{i}+2 \epsilon_{h}\right)$. Here $\epsilon_{h}$ and $\epsilon_{i}$ are the dielectric permittivities of the host and of the MNPs, respectively. The dielectric permittivity of $\mathrm{WO}_{3}$ glass is $\epsilon_{h}=4.2$ at the central operating wavelength of $800 \mathrm{~nm}$. For the dielectric permittivity of the silver MNPs we use the known polynomial approximation [22]: $\epsilon_{i}=\epsilon_{R}+i \epsilon_{I}$, with

$$
\begin{gathered}
\epsilon_{R}=-2.037181 \times 10^{-17} \lambda^{6}+1.183540 \times 10^{-13} \lambda^{5} \\
-2.537882 \cdot 10^{-10} \lambda^{4}+2.430043 \cdot 10^{-7} \lambda^{3} \\
-1.420089 \cdot 10^{-4} \lambda^{2}+8.990214 \cdot 10^{-4} \lambda+8.526028 \\
\epsilon_{I}=-2.327098 \cdot 10^{-17} \lambda^{6}+1.471828 \cdot 10^{-13} \lambda^{5} \\
-3.635520 \cdot 10^{-10} \lambda^{4}+4.530857 \cdot 10^{-7} \lambda^{3} \\
-2.946733 \cdot 10^{-4} \lambda^{2}+9.56229 \cdot 10^{-2} \lambda-11.49465 .
\end{gathered}
$$

For the filling factor of the sample used in the experiment, $f=1.2 \times 10^{-4}$, the group-velocity-dispersion coefficient at $800 \mathrm{~nm}$ is $\beta^{\prime \prime}(\omega) \equiv \partial^{2} k_{\text {eff }} / \partial \omega^{2}=0.0063$ $\mathrm{fs}^{2} / \mu \mathrm{m}$, where $k_{\mathrm{eff}}=n_{\mathrm{eff}}(\omega) \omega / c$ and $n_{\mathrm{eff}}(\omega)=\sqrt{\epsilon_{\mathrm{eff}}}$, and the linear-loss coefficient is $\alpha=\operatorname{Im}\left\{k_{\text {eff }}(\omega)\right\}=$ $1.76 \times 10^{-4} \mu \mathrm{m}^{-1}, c$ being the light velocity in vacuum.

Both the dispersion and losses strongly increase as the plasmon resonance is approached (see particular curves in Ref. 19 ). For the filling factor $f=1.2 \times 10^{-4}$ the dispersion length is few $\mathrm{cm}$, while the effective propagation length and nonlinearity length are few $\mathrm{mm}$, hence 
the dispersion effect is negligible. For spherical MNPs, the effective third-order susceptibility is [23]

$$
\begin{gathered}
\chi_{\mathrm{eff}}^{(3)}=f \frac{\chi_{i}}{|P|^{2} P^{2}}+ \\
+\frac{\chi_{h}\left(1-f+1.6 f \sigma^{2}|\sigma|^{2}+1.2 f \sigma|\sigma|^{2}\right)}{(1-f \sigma)^{2}|1-f \sigma|^{2}} \\
+\frac{\chi_{h}\left(0.4 f \sigma^{3}+3.6 f|\sigma|^{2}+3.6 f \sigma^{2}\right)}{(1-f \sigma)^{2}|1-f \sigma|^{2}}
\end{gathered}
$$

with $P \equiv(1-f \sigma)\left(\epsilon_{i}+2 \epsilon_{h}\right) / 3 \epsilon_{h}$, and $\sigma=\left(\epsilon_{i}-\epsilon_{h}\right) /\left(\epsilon_{i}+\right.$ $\left.2 \epsilon_{h}\right)$, as above. The susceptibility of the host glass is taken to be $\chi_{h}=1.1 \cdot 10^{-21} \mathrm{~m}^{2} / \mathrm{V}^{2}$, which is close to values reported for heavy glasses of the same class [24, which are typically five times higher than the susceptibility of silica glasses. We use the experimentally measured value $\chi_{\mathrm{Ag}}^{(3)}=(-6.3+1.9 i) \cdot 10^{-16} \mathrm{~m}^{2} / \mathrm{V}^{2}[7]$ for the susceptibility of silver nanoparticles. The nonlinear susceptibility of the composite, calculated at the central operation wavelength of $800 \mathrm{~nm}$, is $\chi_{\mathrm{eff}}^{(3)}=(-1.8+0.36 i) \cdot 10$ $-20 \mathrm{~m}^{2} / \mathrm{V}^{2}$. The strong intrinsic nonlinearity of the MNPs tends to make the effective nonlinearity of the composite self-defocusing. Even far from the plasmon resonance, the magnitude and sign of $n_{2}(\omega)$ in the composite strongly depend on the frequency, as shown in Fig. 2. The sign changes to the self-focusing around $1020 \mathrm{~nm}$, similar to what was reported in Ref. [19, which is far away from the spectral range discussed below.

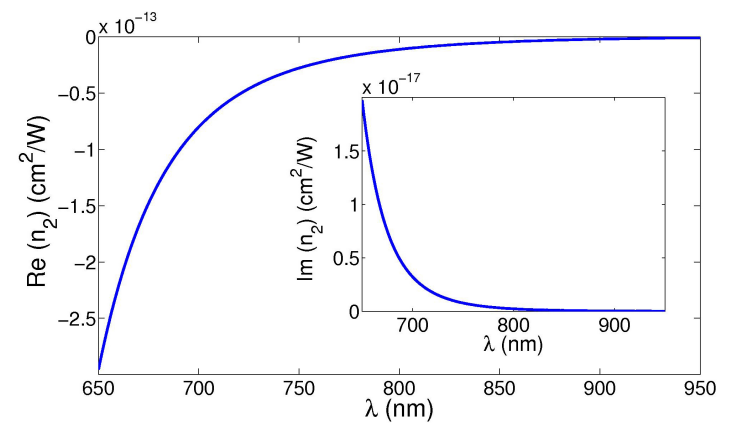

FIG. 2: (Color online) Nonlinear properties of the heavy glass composite with silver nanoparticles. Shown are real and imaginary parts of the nonlinear coefficient, $n_{2}(\omega)=$ $3 \chi_{\text {eff }}^{(3)} /\left(4 c \epsilon_{\text {eff }} \epsilon_{0}\right)$.

To simulate the propagation of the optical pulse through the composite, we use the generalized nonlinear Schrödinger equation 25] for amplitude $A$ of the electric field. This model takes into account the frequency dependence of the nonlinearity, dispersion of all orders, and the temporal delay caused by the stimulated Raman
Scattering (SRS):

$$
\begin{gathered}
\frac{\partial A}{\partial z}=\sum_{m \geq 2} \frac{i^{m+1} \beta_{m}}{m !} \frac{\partial^{m} A}{\partial \tau^{m}}-\frac{\alpha}{2} A \\
+i \gamma(\omega)\left(|A|^{2}-T_{R} \frac{\partial|A|^{2}}{\partial \tau}\right) A .
\end{gathered}
$$

Here $z$ is the propagation distance, $\tau$ the time in the reference frame traveling along with the pump light, $\beta_{m}$ the $m$-th order dispersion coefficient at the central frequency, and $\alpha$ the linear loss. The nonlinear coefficient is given by $\gamma(\omega)=n_{2}(\omega) \omega /\left(c A_{\text {eff }}\right)$, where $n_{2}$ is the nonlinear refractive index of the composite and $A_{\text {eff }}$ the effective mode area. Since the value of the SRS temporal delay in our glass species was not found in literature, it was chosen by fitting to empirical data, $T_{R}=0.32$.

The experimentally measured spectrum in the case of the double propagation $(z=4 \mathrm{~mm})$ of the optical pulse with energy of $1.57 \mathrm{~mJ}$ through the composite containing silver MNPs is presented in Fig. 3 by the solid red curve, together with the spectrum of the same pulse propagated through the host glass, but without nanoparticles (dashed blue curve). Additional curves demonstrate the numerically calculated spectra, including (dot-dashed orange curve) and excluding (dotted blue curve) the Raman term in Eq. (2).

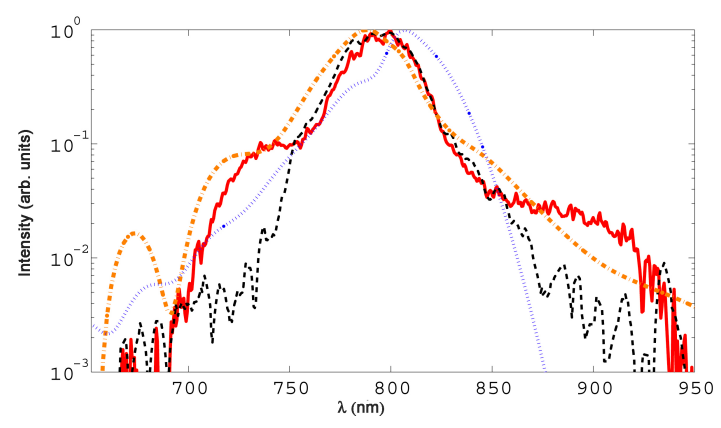

FIG. 3: (Color online) The experimentally measured spectral broadening after the $4 \mathrm{~mm}$-long propagation through the composite sample with and without the nanoparticles (the solid red and dashed black curves, respectively). The numerically evaluated spectrum, with the Raman term taken into regard, is shown by the dot-dashed orange curve, and the one without the Raman term by the dotted blue curve.

In Fig. 3, one can clearly observe an asymmetry in the spectral broadening. The short-wavelength extension of the spectrum originates from the increase of the magnitude of the nonlinearity as the wavelengths decrease toward the plasmon resonance, in accordance with the theoretical prediction [19]. The additional long-wave lower shoulder of the spectrum arises from the SRS shift. Comparing the two numerical curves, one concludes that the inclusion of the SRS effect, which was not considered in the previous analysis reported in Ref. [19], improves the qualitative and quantitative agreement with 
the experimental results. To study the dependence of the spectral broadening on the injected energy, pulses with different values of the peak power were subjected to the double propagation through the sample. The more powerful pulses were injected, the more manifestations of the nonlinear effects in the spectral broadening was observed, as demonstrated in Fig. 4. However, the use of $2 \mathrm{~mJ}$ pulses leads to optical damage of the sample. Additional experiments were performed to establish an optimal propagation length for the pulse broadening. After the single propagation through the glass composite, some initial broadening was observed. The spectral broadening reaches its saturation after the double propagation through the sample $(z=4 \mathrm{~mm})$, with very little change observed after additional propagation cycles.

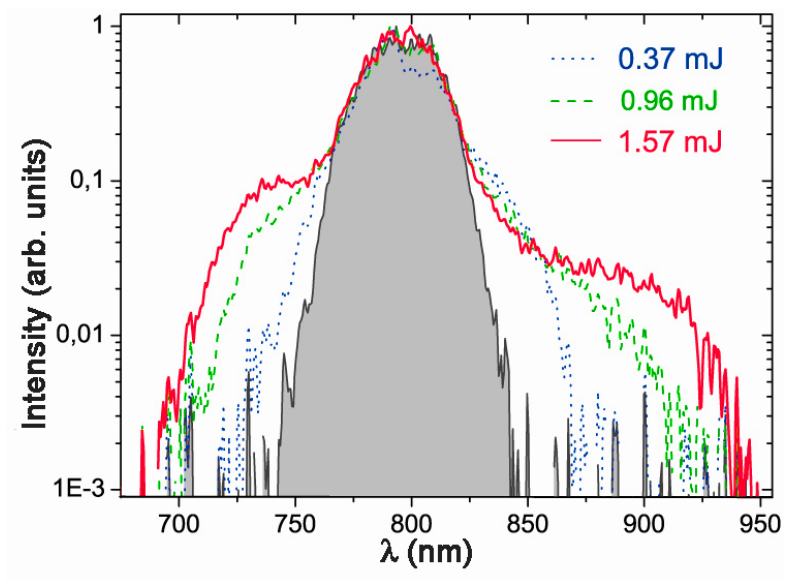

FIG. 4: (Color online) The spectral broadening after the 4 mm long propagation through the composite sample with the nanoparticles, for different energies of the input pulse. The shaded spectrum represents the propagation through the host glass without nanoparticles.

In conclusion, we have demonstrated the asymmetric spectral broadening of the transmitted light pulses due to the presence of silver nanoparticles in $\mathrm{WO}_{3}-\mathrm{SbPO}_{4}-\mathrm{PbO}$ host glasses. The composite exhibits the strong wavelength dependence of the effective nonlinear coefficient, which dominates the shape of the spectral broadening via the self-phase-modulation and leads to the blue spectral upshift. The red wing of the spectrum is induced by the Raman self-frequency shift.

M.S. thank J. M. Caiut for the help with analysis of TEM images.

\section{References}

[1] M. Pelton, J. Aizpurua, G. Bryant, Laser Photonics. Rev. 2, 135 (2008).

[2] G. Schmid, Nanoparticles: From Theory to Application (Wiley Online Library, published online 28 Jan. 2005).

[3] I. Denisyuk and M. Fokina, A Review of High Nanoparticles Concentration Composites: Semiconductor and High Refractive Index Materials ( InTech Publisher, October 2010).

[4] S. Nie, and S. R. Emory, G. Bryant, "Probing Single Molecules and Single Nanoparticles by Surface-Enhanced Raman Scattering," Science 275 1102-1106 (1997).

[5] S. Kuhn, U. Hakanson, L. Rogobete, and V. Sandoghdar, Phys. Rev. Lett. 97, 017402 (2006).

[6] S. Kim, J. Jin, Y. J. Kim, I. Y. Park, Y. Kim, and S. W. Kim, Nature 453, 757-760 (2008).

[7] E. L. Falcão-Filho, C. de Araújo, A. Galembeck, M. M. Oliveira, and A. J. G. Zarbin, J. Opt. Soc. Am. B 22, 2444-2449, (2005).

[8] E. A. Ganeev, A. I. Ryasnyansky, A. L. Stepanov and T. Usmanov, Optical and Quantum Electronics 36, 949 (2004)

[9] D. Rativa, R.E. de Arauju, and A.S.L. Gomes, Opt. Express 16, 19244 (2008)

[10] K. Lance Kelly, Eduardo Coronado, Lin Lin Zhao, and George C. Schatz,The Optical Properties of Metal Nanoparticles: The Influence of Size, Shape, and Dielectric Environment, J. Phys. Chem. B 107, 668-677 (2003)

[11] Dana C. Kohlgraf-Owens and Pieter G. Kik, "Numerical study of surface plasmon enhanced nonlinear absorption and refraction," Optics Express, Vol. 16 Issue 14, pp.10823-10834 (2008).

[12] J. M. Dudley, G. Gentry, and S. Coen, "Supercontinuum generation in photonic crystal fiber," Rev. Mod. Phys.78(4), 1135-1184 (2006).

[13] M. Foster, and A. Gaeta, "Ultra-low threshold supercontinuum generation in sub-wavelength waveguides," Opt. Express 12(14), 3137-3143 (2004).

[14] D. I. Yeom, E. C. Mägi, M. R. E. Lamont, M. A. F. Roelens, L. Fu, and B. J. Eggleton, "Low-threshold supercontinuum generation in highly nonlinear chalcogenide nanowires," Opt. Lett. 33(7), 660-662 (2008).

[15] P. Muhlschlegel, H. J. Eisler, O. J. F. Martin, B. Hecht, and D. W. Pohl, Science 308, 1607 (2005).

[16] S. Besner, A. V. Kabashin, and M. Meunier, Appl. Phys. A 88, 269 (2007).

[17] C. Wang, Y. Fu, Z. Zhou, Y. Cheng, and Z. Xu, Appl. Phys. Lett. 90, 181119, 1-4 (2007).

[18] R. Driben, A. Husakou, and J. Herrmann, Opt. Lett. 34, 2132-2134 (2009)

[19] R. Driben, A. Husakou, and J. Herrmann, Opt. Express 17, 17989-17995 (2009)

[20] R. Driben, and J. Herrmann, Opt. Lett 35, 2529-2531 (2010)

[21] M. Nalin, Y. Messaddeq, S. J. L.Ribeiro, M. Poulain, V. Briois, G. Brunklaus, C. Rosenhahn, B. D. Mosel and H. Eckert, J. Mat Chem.

[22] K. Tanabe, J. Phys. Chem. C 112 15721-15728 (2008) 
[23] J. E. Sipe and R. W. Boyd., Phys. Rev. A 46, 1614-1629, (1992).

[24] F. E. P. dos Santos, C. B. de Araújo, A. S. L. Gomes, K. Fedus, G. Boudebs, D. Manzani, and Y. Messaddeq, Journal of Applied physics. 106, 063507 (2009).

[25] G. P. Agrawal, Nonlinear Fiber Optics, 4th ed., Academic Press, 2007.

\section{Figures captions}

Fig.1 (a)Size distribution silver nanoparticles determined by transmission electron microscopy (TEM). A quasi-spherical geometry as demonstrated in TEM image (inset). (b)The absorption spectrum of the annealed sample.

Fig.2 Nonlinear properties of the heavy glass composite with silver nanoparticles. Shown are real and imaginary parts of the nonlinear coefficient, $n_{2}(\omega)=$ $3 \chi_{\mathrm{eff}}^{(3)} /\left(4 c \epsilon_{\mathrm{eff}} \epsilon_{0}\right)$.

Fig.3 The experimentally measured spectral broadening after the $4 \mathrm{~mm}$ propagation through the composite sample with and without the nanoparticles (the solid red and dashed black curves, respectively). The numerically evaluated spectrum, with the Raman term taken into regard, is shown by the dot-dashed orange curve, and the one without the Raman term by the dotted blue curve.

Fig.4 The spectral broadening after the $4 \mathrm{~mm}$ propagation through the composite sample with the nanoparticles for different energies of the input pulse. The shadded spectrum represents the case of propagation through the host glass without nanoparticles. 